\title{
Acidentes com crianças na educação infantil: percepção e capacitação de professores/cuidadores
}

\author{
Accidents with children in childhood education: \\ perception and training of teachers/caregivers
}

Carolina Matteussi Lino Faculdade de Odontologia de Piracicaba (FOP/UNICAMP), Piracicaba/ SP - Brasil.

Ângela MárCia Fossa Universidade Metodista de Piracicaba (UNIMEP), Piracicaba/ SP - Brasil.

Michele Campagnoli Universidade Metodista de Piracicaba (UNIMEP), Piracicaba/ SP - Brasil.

Mônica Feresini Groppo Universidade Metodista de Piracicaba (UNIMEP), Piracicaba/ SP - Brasil.
Resumo A curiosidade e a vulnerabilidade, decorrentes da idade, tornam as crianças vítimas frequentes de acidentes. Por passarem grande parte do dia na escola, é comum a ocorrência de acidentes nesse ambiente, exigindo dos professores/cuidadores, conhecimentos sobre primeiros socorros. O objetivo do presente estudo foi analisar a percepção desses profissionais quanto aos primeiros socorros e prepará-los para atender a situações de emergência. Tratou-se de um estudo descritivo, de abordagem quali-quantitativo, com aplicação de intervenção, em instituições de educação infantil de um município de pequeno porte, do Estado de São Paulo. Para a coleta dos dados, utilizou-se um questionário para caracterização dos sujeitos e das situações vivenciadas e um para a avaliação dos conhecimentos prévios. Dos sujeitos $(n=87)$, 97\% eram do gênero feminino, com idade média de 42 anos. Os acidentes mais frequentes foram: quedas, sangramento nasal e picada de inseto. Com o intuito de identificar se a capacitação surtiu efeito, o mesmo questionário usado para a avaliação dos conhecimentos prévios foi aplicado. A intervenção mostrou-se eficaz, pois 79,8\% dos indivíduos melhoraram a quantidade de acertos nas questões. A educação continuada e a construção de vínculos e parcerias entre profissionais de saúde locais e professores são necessárias para ampliar a saúde no âmbito escolar.

Palavras-chave: Acidentes escolares. EducaÇão infantil. EduCaÇão em Saúde. Prevenção de acidentes. Saúde escolar.

Abstract The curiosity and the vulnerability, due the age, make children frequent victims of accidents. Because they spend much part of the day in school, it is common to have accidents in this site, requiring teachers/caregivers, knowledge about first aid. The objective of the present study was to analyze the perception of these professionals about first aid and to qualify them to attend emergency situations. This was a descriptive, qualitative-quantitative study, with intervention application, in kindergartens in the small-sized municipality of the state of São Paulo. For the data collection, a questionnaire was used to characterize the subjects and the situations experienced and one for the evaluation of previous knowledge. About the subjects ( $\mathrm{n}=87$ ), 97\% were female, with average age of 42 years. The most frequent accidents were: falls, nasal bleeding and insect stings. In order to identify if the training had 
an effect, the same questionnaire used for the evaluation of previous knowledge was applied. The intervention proved to be effective, since $79.8 \%$ of the individuals improved the number of correct answers to the questions. Continuing education and the links of partnerships between local health professionals and teachers is necessary to increase health in the school setting.

Key-words: School accidents. Child education. Health education. Accidents prevention. School health.

\section{INTRODUÇÃO}

Todo acontecimento não intencional, de origem externa, que resulta em lesões físicas e/ou emocionais, em ambientes domésticos ou sociais, incluindo o âmbito escolar, é denominado acidente. ${ }^{1,2}$

$\mathrm{Na}$ cultura brasileira, os acidentes são considerados eventos inevitáveis, que acontecem por acaso, entretanto, eles podem ser evitados ou controlados, por resultarem de um conjunto de fatores previsíveis. ${ }^{3,4}$

$\mathrm{Na}$ idade pré-escolar, que abrange crianças de 3 a 6 anos de idade, a curiosidade natural, o espírito de independência e o despreparo quanto ao identificar riscos e cuidar de si fazem que elas se exponham a situações que podem levar a acidentes que, em sua maioria, são imperceptíveis, desconhecidos ou menosprezados por seus pais e/ ou responsáveis. ${ }^{2,5}$

Segundo Terassi et al., ${ }^{6}$ as crianças passam em média um terço do seu dia na escola. Sendo assim, acidentes no ambiente escolar são frequentes e podem ocorrer a qualquer momento. Entre os fatores decorrentes destes, encontramos a vulnerabilidade relativa à idade, o ambiente diferente, o descuido dos responsáveis, a inadequação do espaço físico e dos brinquedos e a falta de informação das próprias crianças e cuidadores/professores. ${ }^{2}$

Denomina-se "primeiros socorros" uma série de procedimentos prestados após al- gum acidente, com a finalidade de preservar a vida em uma situação de risco iminente e/ ou em condições de urgência e emergência, evitando o agravamento das condições gerais da vítima até a chegada de assistência qualificada.,

$\mathrm{O}$ auxílio às vítimas em situações de urgência e emergência ainda é um tema desconhecido para a maioria das pessoas. Esse fato faz que, perante um acidente, o socorrista haja por impulso, podendo causar danos irreparáveis para a vítima em questão.,

No espaço escolar, os acidentes são uma preocupação constante, sendo necessário que os professores/cuidadores saibam como evitá-los e como realizar os primeiros socorros, impedindo assim complicações decorrentes de procedimentos inadequados. ${ }^{5}$ Sendo assim, o decreto $n^{\circ}$. 6.286, instituído pelo Governo Federal no ano de 2007, deu origem ao Programa Saúde na Escola (PSE), com o intuito de promover a saúde e prevenir agravos e doenças, por meio de um trabalho conjunto entre escola e unidade de saúde. ${ }^{10}$

Esse programa tem por finalidade a instituição de políticas de promoção e melhoria da qualidade de vida de crianças, adolescentes, jovens e adultos que utilizam a educação pública, além de envolver os professores e funcionários dessas instituições. ${ }^{10}$

Diante do exposto, surgiu a necessidade de melhor compreender como os professores e cuidadores agem perante os acidentes 
escolares e quão preparados encontram-se para prestar os primeiros socorros, caso necessário. Dessa forma, o presente estudo teve como objetivo analisar a percepção dos professores e cuidadores das escolas de ensino infantil, buscando socializar informações e prepará-los para atender a situações de emergências com crianças.

\section{Métodos}

Estudo descritivo, com abordagem quali-quantitativa e intervencionista, realizado em instituições de educação infantil, que atendem crianças a partir de três meses até 5 anos de idade, vinculadas à Secretaria $\mathrm{Mu}$ nicipal de Educação, em um município de pequeno porte do Estado de São Paulo, no período de maio a junho de 2016.

Os sujeitos foram professores, professores auxiliares infantis (P.A.I.), auxiliares de educação infantil (A.E.I.) e demais funcionários que aceitaram participar da pesquisa. Os participantes que aceitaram participar assinaram o Termo de Consentimento Livre e Esclarecido (TCLE), de acordo com o estabelecido na Resolução 466/12 do Conselho Nacional de Saúde.

A pesquisa foi aprovada pelo Comitê de Ética em Pesquisa da Universidade Metodista de Piracicaba (parecer 30/2016). Não há conflito de interesse em relação aos métodos utilizados como parte da investigação ou interesse financeiro dos pesquisadores.

Foram excluídos profissionais que não aceitaram participar da pesquisa, que estavam de licença e/ou férias no período da coleta dos dados e os que não preencheram os questionários de avaliação (dois sujeitos).
Os dados foram obtidos por meio da aplicação de dois instrumentos, sendo um semiestruturado e um estruturado, elaborados pela pesquisadora. O primeiro instrumento, um questionário semiestruturado, foi composto por questões de caracterização dos sujeitos, dos acidentes presenciados e com um campo para relato das situações nas quais a aplicação dos primeiros socorros foi necessária. O segundo instrumento, um questionário estruturado, apresentava 12 questões de múltipla escolha, com situações que envolviam acidentes no âmbito escolar, como obstrução de vias aéreas, febre, epistaxe, convulsão, desmaio, corpo estranho, ferimentos, queimadura, intoxicação e quebra/avulsão de dentes.

Após a aplicação dos questionários, houve uma capacitação, com duração de uma hora, de modo a agregar conhecimento das medidas de primeiros socorros a serem tomadas em casos de acidentes com crianças. Posteriormente, com a utilização do segundo instrumento, avaliamos as informações assimiladas durante a intervenção.

A coleta dos dados e a capacitação foram realizadas durante a Hora de Trabalho Pedagógico Coletivo (HTPC), das 17h30 às 19h30, pois todos estavam reunidos e não havia mais expediente com as crianças. Os encontros foram registrados por meio da elaboração de um diário de bordo, a fim de documentar os principais questionamentos e ocorrências durante as capacitações.

Os dados quantitativos foram tabulados no programa Microsoft Excel $2010^{\circledR}$ e analisados no programa EPI Info ${ }^{\circledR}$ versão 7. Os dados qualitativos foram analisados com base no conteúdo e foram classificados em categorias. 


\section{Resultados}

Ao todo, participaram cinco instituições de educação infantil, sendo três municipais e duas filantrópicas, totalizando 87 sujeitos. Destes, $97 \%$ eram do sexo feminino e $3 \%$ do masculino, com faixa etária entre $22 \mathrm{e}$ 59 anos de idade, sendo 40 a 49 anos a faixa etária mais frequente. Quanto às funções, a grande maioria foi composta por auxiliares de educação infantil (A.E.I.), com 36\%, seguido por professores (33\%) e professor au- xiliar infantil (P.A.I.), com 15\% dos sujeitos. Entre a classe "outros", compreende-se merendeira, servente, coordenadora pedagógica, oficial (I e II), diretora, gerente administrativo e função não informada (Tabela 1). No que diz respeito à escolaridade, $47 \%$ possuíam ensino superior, seguidos por $36 \%$ que tinham o ensino médio. Quanto ao tempo de experiência na instituição, a média foi de cinco anos, sendo o tempo mínimo de um mês e o máximo de 29 anos (desvio padrão 5,0).

TABELA 1 - Características demográficas de 87 profissionais de cinco instituições de ensino infantil em um município de pequeno porte, 2016.

\begin{tabular}{|c|c|c|c|}
\hline Variáveis & Descrição & Frequência & $\%$ \\
\hline \multirow[t]{2}{*}{ Sexo } & Feminino & 84 & 97 \\
\hline & Masculino & 3 & 3 \\
\hline \multirow[t]{5}{*}{ Idade } & $20-29$ anos & 9 & 10 \\
\hline & 30-39 anos & 27 & 31 \\
\hline & $40-49$ anos & 30 & 34 \\
\hline & $50-59$ anos & 21 & 24 \\
\hline & Média \pm desvio padrão $(42 \pm 9,02)$ & & \\
\hline \multirow[t]{5}{*}{ Escolaridade } & Ensino fundamental & 2 & 2 \\
\hline & Ensino médio & 31 & 36 \\
\hline & Ensino superior & 41 & 47 \\
\hline & Pós-graduação & 12 & 14 \\
\hline & Não informado & 1 & 1 \\
\hline \multirow[t]{4}{*}{ Função } & Auxiliar de educação infantil (A.E.I.) & 31 & 36 \\
\hline & Professor & 29 & 33 \\
\hline & Professor auxiliar infantil (P.A.I.) & 13 & 15 \\
\hline & Outros & 14 & 16 \\
\hline \multirow[t]{7}{*}{ Tempo na instituição } & Menos de 2 anos & 22 & 25 \\
\hline & 2 a 5 anos & 26 & 30 \\
\hline & 6 a 10 anos & 29 & 33 \\
\hline & 11 a 15 anos & 3 & 3 \\
\hline & 16 anos ou mais & 5 & 6 \\
\hline & Não informado & 2 & 2 \\
\hline & \multicolumn{2}{|c|}{ Média \pm desvio padrão $(5$ anos $\pm 5,0)$} & \\
\hline
\end{tabular}


Quanto ao tempo na instituição, 33\% possuíam de seis a dez anos de atuação, $30 \%$ de dois a cinco anos e $25 \%$ tinham um tempo inferior a dois anos. Os sujeitos que apresentaram um tempo igual ou maior a 16 anos totalizaram $6 \%$.

As crianças estão expostas a acidentes, inclusive no âmbito escolar, por passar a maior parte do dia nesse local. Dos sujeitos deste estudo, 58,6\% presenciaram algum tipo de acidente na escola.

As quedas foram os acidentes mais comuns, seguido de sangramento nasal e picada de inseto, conforme Figura 1. Dentro da classe "Outros", compreendem-se "criança presa na gangorra", "engasgo com comida", "queimaduras", "febre", "mordida de outra criança", "mordedura de animal", "intoxicação" e "asfixia".

Os sujeitos compartilharam experiências de acidentes com crianças, nos quais foi necessária a aplicação de primeiros socorros.

\begin{abstract}
"A princípio foi um susto (referente à fratura), mas depois com calma conseguimos imobilizar o braço usando uma placa de isopor (é o que tínhamos por perto)." (Q1)
\end{abstract}

"Consegui administrar a ocorrência, coloquei gelo no local da batida (referente à queda) e fiquei observando a criança com mais ênfase." (Q2)

"Foi uma queda, machucou a testa e ficou um galo.” (Q3)

"Corpo estranho (nariz): acompanhei a criança até o pronto-socorro.” (Q4)

Quanto ao procedimento a ser adotado após o acidente presenciado, $47 \%$ dos sujeitos relataram saber o que fazer perante a situação. Durante a análise dos procedimentos adotados e relatados pelos participantes, foi possível identificar que, em algumas situações onde foi necessária a aplicação de primeiros socorros em crianças, os sujeitos

FIGURA 1 - Tipos de acidentes infantis presenciados com maior frequência, por profissionais, cuidadores de crianças, em instituições de ensino infantil, 2016.

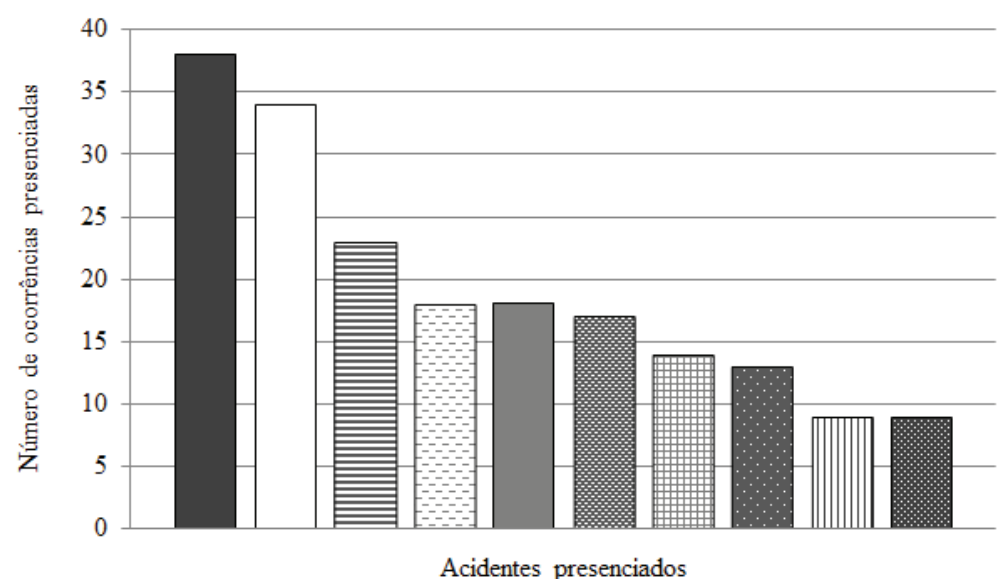

Sáude em Revista

Acidentes com crianças na educação infantil: percepção e capacitação de professores/cuidadores
- Queda

$\square$ Sangramento nasal

घPicada inseto

$\square$ Cortes

$\square$ Corpo estranho

圆Crise convulsiva/epilética

$\boxplus$ Queda/avul são dente

Fraturas

mDesmaio

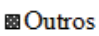


agiram de forma diferente do preconizado na literatura. Ainda, 46\% não informaram se "souberam o que fazer" perante os acidentes observados. Acredita-se que esse alto índice tenha ocorrido pela insegurança de poder ter tomado uma atitude inadequada.

A avaliação do conhecimento prévio dos professores e/ou cuidadores apontou a desatualização perante a aplicação de primeiros socorros, além de demonstrar que o conhecimento popular, aliado com essa ausência de informação, são fatores que influenciam nas condutas de primeiros socorros adequados às vítimas de acidentes escolares.

Os relatos a seguir evidenciaram que alguns procedimentos adotados perante a necessidade de primeiros socorros indicaram desconhecimento e falta de capacitação dos profissionais das instituições, além de sentimentos de despreparo e insegurança.

\footnotetext{
"Foi muito tenso, mas procurei manter a calma e ajuda-la, a voltar ao normal, sacudi ela, assoprei seu rosto (referindo-se ao desmaio)." (Q5)

“Assustei (referindo-se à convulsão), mas já estava preparada pelos conselhos da mãe: abanando, deitando-o, deixar ele bem calmo, água etc.” (Q6)

"Queda sempre foi simples, bateu a cabeça eu sempre fazia compressa com gelo (...). Sangramento nasal, sentei a criança com a cabeça levemente inclinada para cima." (Q7)

"No caso da epilepsia, foi muito difícil pois não sabia como socorrer a criança.” (Q8)

"Muita preocupação, fiquei muito nervosa, com medo e assustada." (Q9)
}

Quanto às dificuldades encontradas durante a realização dos primeiros socorros, identificamos o fato de o sujeito não saber se está agindo corretamente perante a necessidade de aplicação dos primeiros socorros e a ausência de treinamento sobre o assunto.

\begin{abstract}
"A insegurança e o despreparo por estar tomando a atitude mais acertada são dificuldades existentes." (Q10)

"Foi complicado em algumas situações que não sabia como solucionar, que providências tomar.” (Q11)

“A maior dificuldade que pude encontrar é de estar agindo de forma correta." (Q12)

"Falta de treinamento e diálogo." (Q4)

"Não ter conhecimento técnico para socorrer, assim foi feito o que tinha conhecimento." (Q13)
\end{abstract}

Com o intuito de agregar conhecimentos quanto a acidentes na infância e aplicação de primeiros socorros, foram feitas capacitações com os sujeitos, no próprio ambiente das instituições de ensino infantil. Foram realizados quatro encontros, com duração média de uma hora, e aproximadamente 22 participantes por dia. $\mathrm{O}$ material didático foram slides com as situações abordadas e uma boneca, para a demonstração de técnicas e manobras.

Nessas capacitações, abordaram-se os acidentes mais comuns de acordo com cada faixa etária e como prestar os primeiros socorros no caso de obstrução das vias aéreas, febre, epistaxe, crise convulsiva/epilética, desmaio, traumas (principalmente o encefálico), fraturas, ferimentos, corpos estranhos, intoxicação, mordedura de animais, acidentes com animais peçonhentos, queimaduras e quebra/avulsão de dentes.

A fim de identificar se a intervenção atingiu o objetivo proposto, após a capacitação, o mesmo questionário foi aplicado aos sujeitos. A Figura 2 refere-se ao número absoluto de questões respondidas corretamente antes e após a intervenção. 
FIGURA 2 - Mediana das questões respondidas corretamente pelos profissionais cuidadores de crianças em instituições de ensino infantil (máximo possível = 12) antes e após a intervenção.
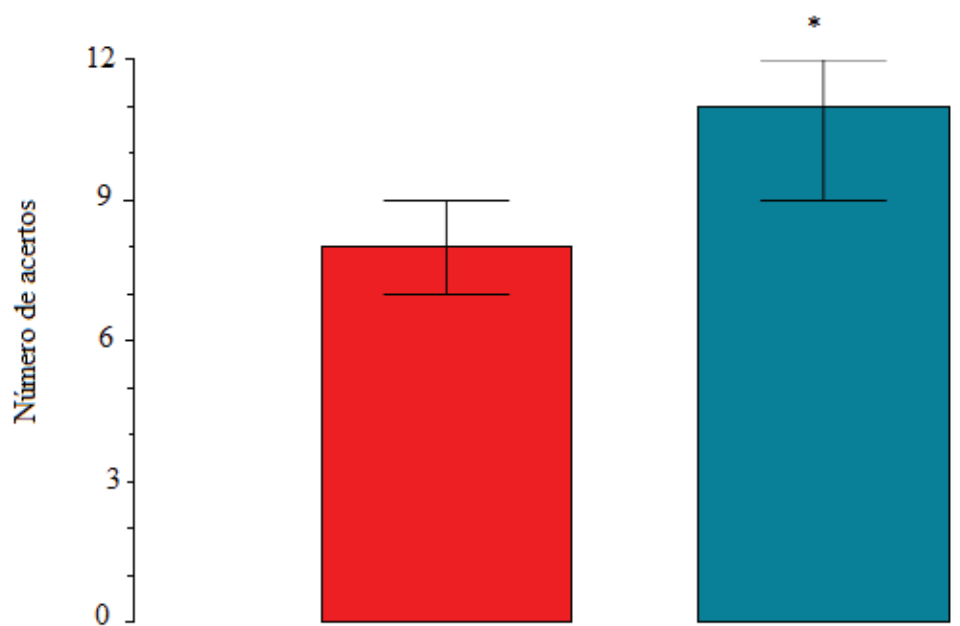

Antes

Depois

${ }^{\star}$ Diferenças estatisticamente significantes entre os momentos (teste de Wilcoxon, $\mathrm{p}<0,0001$ ).

Antes da realização da intervenção, os acertos apresentaram mediana 8 e moda 9 (29 sujeitos). Após a intervenção, a mediana foi 11 , com moda 12 (35 sujeitos).

Foi possível observar que houve um aumento significativo $(\mathrm{p}<0,0001)$ do número de acertos referentes ao questionário após a aplicação da intervenção (capacitação), mostrando que esta foi eficaz. Melhoraram a quantidade de acertos após a intervenção,
$79,8 \%$ dos indivíduos, sendo que 18\% mantiveram a mesma quantidade de acerto.

Identificou-se que as questões número 2 (referente a cuidados perante um quadro de febre) e 11 (referente a cuidados perante avulsão dentária) apresentaram menor porcentagem de acertos antes da capacitação, com 25\% e $24 \%$ de acertos, conforme demonstrado na Figura 3. Após a intervenção, o percentual de acerto elevou-se para $53 \%$ e $47 \%$.

FIGURA 3 - Índice de acerto dos sujeitos cuidadores de crianças em instituições de ensino infantil, segundo procedimentos assinalados na avaliação antes e após a capacitação (n=87)

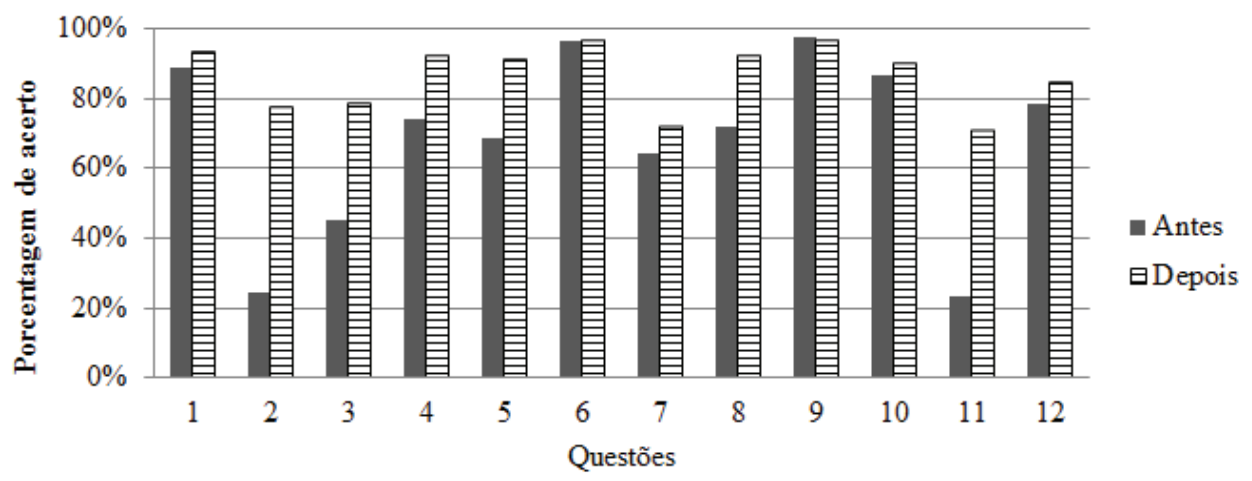




\section{Discussão}

Ressalta-se que a considerável prevalência de indivíduos do gênero feminino dentro da amostra não se limita somente a este estudo, sendo que outros autores encontraram $86,6 \%{ }^{11}$ e $100 \%^{2}$ do gênero feminino entre professores de educação infantil.

A presença masculina nessas instituições, como professores e/ou cuidadores, ainda é pequena, devido à presença de preconceito e desconfiança, uma vez que essas funções são culturalmente atribuídas ao gênero feminino e envolvem procedimentos de cuidados corporais das crianças, ocasionando conflitos e tornando-os suspeitos de possíveis abusos. ${ }^{12,13}$

A faixa etária encontrada neste estudo é divergente da literatura, onde o encontrado foi 31 a 40 anos $(39,3 \%) .^{2}$ Segundo o Instituto Brasileiro de Geografia e Estatística (IBGE), a população estimada no município em que este estudo foi realizado, no ano de 2016, era de 22.914 habitantes. ${ }^{14}$ Por se tratar de um município de pequeno porte, sua demanda e, por conseguinte, a abertura de poucos processos seletivos, podem justificar essa diferença entre as faixas etárias da literatura das identificadas no presente estudo.

Durante a coleta de dados e a realização das intervenções, foi possível identificar que o tempo de trabalho na instituição melhora a prática de cuidados com as crianças, auxiliando nos cuidados em casos de acidentes. Essa percepção corroborou com o encontrado por Neto et al., ${ }^{15}$ que referem que o tempo de trabalho na instituição auxilia nos cuidados com as crianças, devido ao conhecimento das rotinas da instituição pelos profissionais.
Durante a intervenção, pôde-se perceber que quanto maior a escolaridade dos sujeitos, tanto maiores foram os conhecimentos para a aplicação dos primeiros socorros. $\mathrm{Na}$ literatura, não foi possível encontrar estudos que relacionassem a escolaridade dos professores e/ou cuidadores com a realização dos primeiros socorros em casos de acidentes escolares.

O tipo de acidente presenciado com maior frequência pelos sujeitos deste estudo foi a queda, corroborando com a literatura. Pesquisas realizadas ${ }^{15,16,17,18,19}$ indicaram que a faixa etária de 1 a 4 anos é a que apresenta maior índice de quedas ${ }^{16}$ e que as quedas são frequentes nas creches, podendo variar de $31,5 \%{ }^{15}$ a $40,6 \%{ }^{19}$ das ocorrências de acidentes com crianças. Esse tipo de acidente ocorre com maior frequência, devido à vulnerabilidade ocasionada pela imaturidade, curiosidade, intenso crescimento e desenvolvimento ${ }^{15}$ e, também, por estarem realizando atividades lúdicas em ambientes diferentes, como parque, por exemplo.

O conhecimento das experiências dos professores e cuidadores, quanto ao atendimento a ocorrências de acidentes é relevante, uma vez que esses profissionais são responsáveis pelo processo de aprendizagem e, também, pelos cuidados em casos de acidentes. $^{21}$

Diante da ocorrência de acidentes em instituições de ensino infantil, os professores e cuidadores buscam prestar os primeiros socorros, entretanto, o sentimento de despreparo e insegurança, e o medo de agir de forma inadequada se fazem presentes. ${ }^{3} \mathrm{E}$ comum que professores/cuidadores adotem procedimentos inadequados, tendo em vista a falta de conhecimento, habilidades e in- 
formações quanto às medidas corretas. ${ }^{11}$ As reações individuais serão diversas, podendo variar entre saber as medidas a serem tomadas e ficar paralisado perante a situação presenciada, ou não saber o que fazer, mas possuir coragem para a realização dos primeiros socorros (mesmo podendo agir de maneira equivocada). ${ }^{22}$

Os resultados apontaram que os professores e cuidadores possuíam um conhecimento prévio empírico sobre primeiros socorros, entretanto, esses conhecimentos estavam associados às crenças populares. A dúvida mais frequente durante as capacitações foi relacionada às medidas a serem tomadas perante uma criança com crise convulsiva. A crença popular de que o socorrista precisa "puxar a língua" da vítima, para evitar que ela "afogue" predominou em todas as instituições e corroborou com a literatura, na qual $65,3 \%$ dos entrevistados afirmaram que abririam a boca da criança em crise convulsiva, evitando que a vítima enrolasse a língua. ${ }^{20} \mathrm{O}$ grande desafio encontrado nas intervenções foi a desconstrução dessas crenças, e a promoção de conceitos adequados sobre primeiros socorros, uma vez que a tomada de medidas corretas minimiza os riscos às vítimas.
A capacitação e a educação continuada sobre primeiros socorros são necessárias, para que professores não entrem em pânico nas situações nas quais esses procedimentos são aplicáveis. ${ }^{2}$

\section{CONSIDERAÇões Finais}

A ausência de programas de capacitação e educação em saúde para professores, cuidadores e demais profissionais atuantes nas instituições de ensino infantil faz que essa população se sinta insegura e despreparada perante situações de emergência. Mediante isso, a percepção dos sujeitos avaliados foi "saber o que fazer" com embasamento no conhecimento popular, não condizentes com a literatura, que foram trabalhados durante a capacitação.

Nesse sentido, tendo como base o Programa Saúde na Escola, ${ }^{10}$ sugerimos uma aproximação entre os serviços/profissionais de saúde local e as instituições de educação infantil, com políticas de prevenção e elaboração de cronogramas de atividades que envolvam essas instituições, visando à construção de um vínculo e a educação continuada dos professores, cuidadores e demais profissionais, para ampliar a saúde no âmbito escolar.

\section{REFERÊNCIAS}

1. Brasil. Ministério da Saúde. Manual de Primeiros Socorros. Rio de Janeiro: Fundação Oswaldo Cruz; 2003.

2. Siebeneichler AEM, Hahn GV. Professores da pré-escola e o agir em situações de emergência. Rev. Destaques Acadêmicos. 2014; 6 (3): 139-147.

3. Silvani CB, Gomes GC, Sousa LD, Souza JL. Prevenção de acidentes em uma instituição de educação infantil: o conhecimento das cuidadoras. Rev. Enferm. UERJ, 2008 ; 16 (2): 200-205.

4. Tinoco VA, Reis MMT, Freitas LN. O enfermeiro promovendo saúde como educador escolar: atuando em primeiros socorros. Rev. Transformar. 2014; 1 (6): 104-113.

5. Brolezi EA. Orientações de primeiros socorros em urgência na escola. Saúde em foco. 2014: 111-123. 
6. Terassi M., Borges AKPG, Garanhani ML, Martins EA. A percepção de crianças do ensino fundamental sobre parada cardiorrespiratória. Semin., Ciênc. Biol. Saúde. 2015; 36 (1): 99-108.

7. Corpo de Bombeiros Militar do Estado do Paraná - CBMPR. Primeiros socorros. Curitiba, Paraná [acesso 2017 jul. 1]. Disponível em http://www.bombeiros.pr.gov.br/modules/conteudo/ conteudo.php? conteudo $=87$

8. Pereira KC, Paulino JR, Saltarelli RMF, Carvalho AMP, Santos RB, Silveira TVL et al. A construção do conhecimento sobre prevenção de acidentes e primeiros socorros junto ao público leigo. Revista de Enfermagem do Centro-Oeste Mineiro. 2015; 5 (1): 1478-1485.

9. Veronese AM, Oliveira DLLC, RIM, Nast K. Oficinas de primeiros socorros: relato de experiência. Rev. Gaúcha Enferm. 2010, mar.; 31 (1): 179-182.

10. Brasil. Ministério da Saúde. Caderno do gestor do PSE. Ministério da Saúde. Ministério da Educação. Brasília: Ministério da Saúde, 2015: 68.

11. Carvalho LS, Alarcão ALC, Barroso PD, Meireles GOAB. A abordagem de primeiros socorros realizada pelos professores em uma unidade de ensino estadual em Anápolis-GO. Ensaios Cienc., Cienc. Biol. Agrar. Saúde. 2014; 18 (1): 25-30.

12. Ramos J., Xavier MC. A presença de educadores do sexo masculino na educação e cuidado com crianças pequenas. In: Fazendo gênero 9 diásporas, diversidades, deslocamentos, 2010. Santa Catarina.

13. Monteiro MK, Altmann H. Homens na educação infantil: olhares de suspeita e tentativa de segregação. Cadernos de Pesquisa. 2014; 44 (153), 720-741.

14. Instituto Brasileiro de Geografia e Estatística IBGE. Informações estatísticas Cidades. 2016 [acesso 2016 nov. 8]. Disponível em: http://cidades.ibge.gov.br/xtras/perfil.php?codmun=352140

15. Neto CM, Alves NC, Paes MSL. Risco de acidentes na infância em uma creche comunitária de Ipatinga/MG. Revista Enfermagem Integrada. 2010; 3 (1): 381-394.

16. Poll MA, Weiller TH, Engel RH, Borges TAP, Rios AO, Carpes VAC. Quedas de crianças e adolescentes: prevenindo agravos por meio de educação em saúde. Rev. Enferm. UFSM, 2013; 3: 589-598.

17. Moraes MCL, Silva EBC. Estudo sobre os acidentes na infância em duas creches públicas do município de São Paulo. Revista Areté| Revista Amazônica de Ensino de Ciências. 2017; 7 (14): 124-134.

18. Dias MP, de Carvalho MD, Joventino ES, Uchoa JL, Tavares MC, Morais LA, Ximenes LB. Identificação dos fatores de risco para acidentes na primeira infância no contexto creche. Revista de APS. 2013; 16(1): 20-26.

19. Lima RPD, Ximenes LB, Joventino ES, Vieira LJEDS, Oriá MOB. Principais causas de acidentes domésticos em crianças: um estudo descritivo-exploratório. On-line braz. j. nurs. 2008; 7 (3).

20. Lunard MS. Educação em epilepsia para profissionais da educação [dissertação]. Florianópolis: Universidade Federal de Santa Catarina; 2012.

21. Coelho LCA, Silva LRC. Formação docente, educação e prevenção de acidentes. In: Congresso Nacional de Educação, 2011. Curitiba. Anais; Curitiba EDUCERE, 2011: 7922-7933.

22. Rossa JC.; Ferreira GML. Nível de conhecimento de primeiros socorros de todos os professores e funcionários administrativos de uma escola municipal de Medianeira - PR. Faculdade de Ensino Superior de São Miguel do Iguaçu, 2010. 
DADO(S) AUTOR(ES)

\section{CAROLINA MATTEUSSI LINO}

Enfermeira, Mestranda do Programa de Pós-Graduação em Odontologia, área de concentração Saúde Coletiva, pela Faculdade de Odontologia de Piracicaba (FOP/UNICAMP), Piracicaba/SP - Brasil. carolina.matteussi@gmail.com

\section{ÂNGELA MÁRCIA FOSSA}

Enfermeira, Mestre em Educação pela Universidade Metodista de Piracicaba (UNIMEP). Docente da Faculdade de Ciências da Saúde (FACIS) da Universidade Metodista de Piracicaba (UNIMEP), Piracicaba/SP - Brasil. amfossa@uol.com.br

\section{MICHELE CAMPAGNOLI}

Enfermeira, Mestre em Educação pela Universidade Estadual de Campinas (UNICAMP). Docente da Faculdade de Ciências da Saúde (FACIS) Universidade Metodista de Piracicaba (UNIMEP), Piracicaba/SP - Brasil. michelecampagnoli@yahoo.com.br

\section{MÔNICA FERESINI GROPPO}

Enfermeira, Doutoranda do Programa de Pós-graduação em Odontologia pela Faculdade de Odontologia de Piracicaba (FOP/UNICAMP). Docente da Faculdade de Ciências da Saúde (FACIS) da Universidade Metodista de Piracicaba (UNIMEP), Piracicaba/SP - Brasil. mofgroppo@yahoo.com.br

Submissão em: 09/11/2017

Aceite em: 30/01/2018 